\title{
Truncation of ADAMTS13 by Plasmin Enhances Its Activity in Plasma
}

\author{
Chantal C. Clark ${ }^{1, *}$ Mirjam M. Mebius ${ }^{1,2, *}$ Steven de Maat ${ }^{1} \quad$ Aloysius G. M. Tielens 2,3 \\ Philip G. de Groot ${ }^{1}$ Rolf T. Urbanus ${ }^{1}$ Rob Fijnheer ${ }^{1}$ Bouke P.C. Hazenberg ${ }^{4}$ Jaap J. van Hellemond ${ }^{2}$ \\ Coen Maas ${ }^{1}$
}

\footnotetext{
${ }^{1}$ Department for Clinical Chemistry and Haematology, University Medical Center Utrecht, Utrecht University, Utrecht, The Netherlands

2 Department of Medical Microbiology and Infectious Diseases, Erasmus University Medical Center, Rotterdam, The Netherlands

${ }^{3}$ Department for Biochemistry and Cell Biology, Faculty Veterinary Medicine, Utrecht University, Utrecht, The Netherlands

${ }^{4}$ Department of Rheumatology and Clinical Immunology, University Medical Center Groningen, University of Groningen, Groningen, The Netherlands
}



Address for correspondence Dr. Coen Maas, Department of Clinical Chemistry and Haematology, University Medical Center Utrecht, Utrecht University, Heidelberglaan 100, Room G.03.550, 3584 CX, Utrecht, The Netherlands (e-mail: cmaas4@umcutrecht.nl).

Thromb Haemost 2018;118:471-479.

\begin{abstract}
Keywords

- ADAMTS13

- von willebrand factor

- plasmin

ADAMTS13 (a disintegrin and metalloproteinase with a thrombospondin type 1 motif, member 13) cleaves von Willebrand Factor (VWF) multimers to control their thrombogenicity. The fibrinolytic enzyme plasmin can cleave VWF in a similar manner. However, plasmin can also cleave ADAMTS13, which ultimately inactivates it. This leaves the overall role of plasmin in primary haemostasis uncertain.

We investigated the combined molecular effects of plasmin on VWF and ADAMTS13. We first identified that plasmin destroys FRETS-VWF73 substrate by cleaving the ADAMTS13 binding region in a buffered system. We next investigated how plasmin affects both VWF and ADAMTS13 under static conditions in plasma by western blotting. We found that globular VWF is largely protected from plasmin cleavage. However, ADAMTS13 is rapidly cleaved under these conditions, suggesting inactivation. Surprisingly, we observed that plasmin enhances ADAMTS13 activity in a modified two-stage FRETS-VWF73 assay that protects FRETS-VWF73 substrate from degradation. In direct binding studies under the same conditions, we found that plasmin generates multiple C-terminally truncated forms of ADAMTS13 with VWF-binding capacity. In an effort to seek evidence for this mechanism in vivo, we analysed plasma from patients with systemic amyloidosis, which is hallmarked by a hyperfibrinolytic state. We found that their plasma contained increased levels of C-terminally truncated forms of ADAMTS13, which correlated with their hyperfibrinolytic state.

We propose that truncation of ADAMTS13 by plasmin abolishes intramolecular selfassociation, which improves interaction with unfolded VWF.
\end{abstract}

* Chantal C. Clark and Mirjam M. Mebius contributed equally.

received

November 2, 2017

accepted after revision

December 21, 2017
Copyright (c) 2018 Schattauer

DOI https://doi.org/ 10.1055/s-0038-1627460. ISSN 0340-6245. 


\section{Introduction}

The metalloproteinase ADAMTS13 (a disintegrin and metalloproteinase with a thrombospondin type 1 motif, member 13) cleaves von Willebrand Factor (VWF) multimers to control their thrombogenicity. As a result, low or absent ADAMTS13 activity is associated with thrombotic thrombocytopenic purpura (TTP), a rare form of thrombotic microangiopathy. ${ }^{1}$ TTP attacks are accompanied by plasminogen activation. ${ }^{2}$ We previously found preclinical evidence for a protective role of plasmin in TTP: it can degrade platelet-VWF complexes and stimulation of plasmin activity has therapeutic value in a mouse model for TTP. ${ }^{2}$ However, unrestricted exposure of ADAMTS13 to plasmin in a buffered system destroys its activity. ${ }^{3}$ These seemingly opposite functions of plasmin leave its overall contribution in physiological primary haemostasis unclear. In the present study, we aimed to obtain insight into the effects that plasmin simultaneously exerts on both VWF and ADAMTS13 under static conditions in plasma.

A monoclonal antibody mapping study previously showed that plasmin cleaves VWF into three fragments. This indicates the presence of two distinct cleavage sites, rather than random cleavage after 1 of the 108 lysine residues ( $4 \%$ of all residues) in a VWF monomer. ${ }^{4}$ One of the plasmin cleavage sites was mapped to a region within VWF A2 domain (1476-1643 numbering includes pro-peptide), where the ADAMTS13 cleavage site is also located (1605-1606 numbering includes pro-peptide). ${ }^{4}$ This helps explain our finding that plasmin cleaves VWF in a conformation-dependent manner similar to ADAMTS13. ${ }^{2}$ The diagnostic ADAMTS13 activity assay (FRETS-VWF73 assay) uses a synthetic peptide based on the VWF A2 domain, including the ADAMTS13 cleavage site, flanked by a fluorophore and quencher (-Fig. 1A). ${ }^{5}$ The long sequence that follows the quencher is required for ADAMTS13 binding, and subsequent cleavage. $^{5}$ In our study, we first investigated the effect of plasmin on FRETS-VWF73 substrate and VWF in plasma. Subsequently, we investigated the effect of plasmin on ADAMTS13 activity and structure.

\section{Material and Methods}

\section{Reagents}

The following reagents were used: normal pooled plateletpoor citrated plasma (hereafter called plasma; 50 healthy donors, Sanquin, the Netherlands); FRETS-VWF73 substrate (AnaSpec, Fremont, California, United States); plasmin substrate (I-1390; Bachem, Bubendorf, Switzerland); rabbit polyclonal anti-VWF (A0082; DAKO, Carpinteria, California,
A
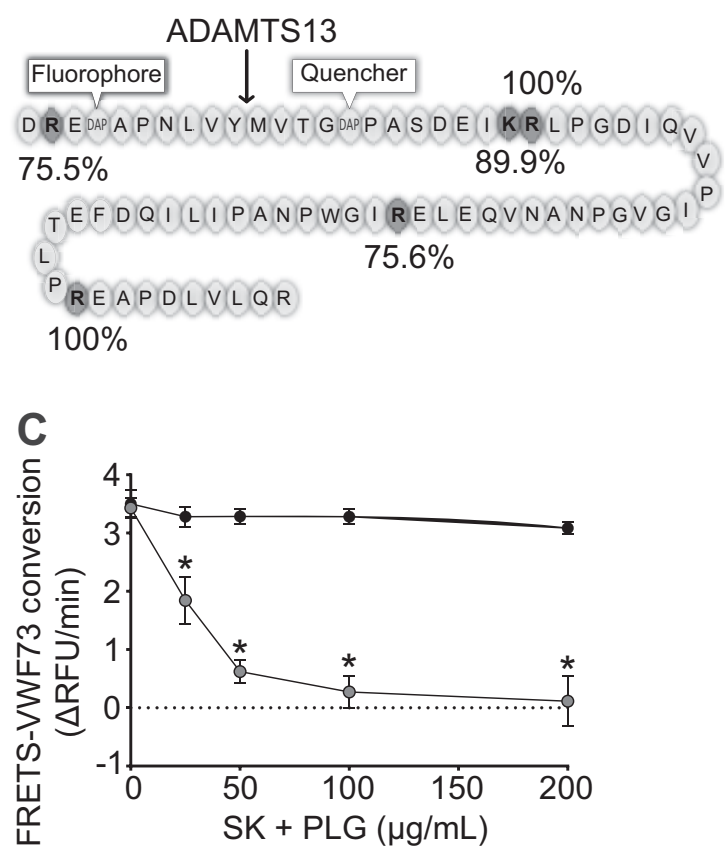

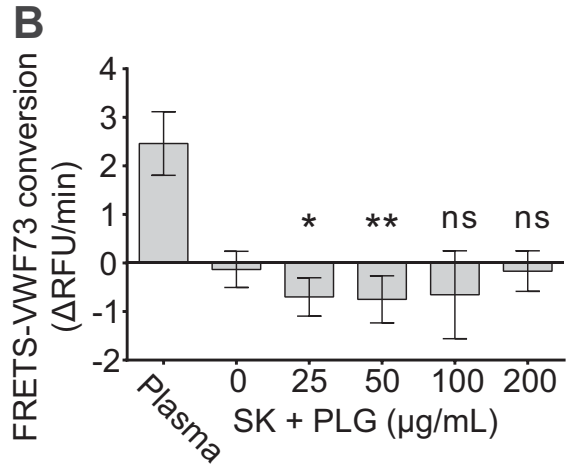

Fig. 1 Plasmin cleaves the ADAMTS13 (a disintegrin and metalloproteinase with a thrombospondin type 1 motif, member 13) binding tail of FRETS-VWF73 substrate in buffer. (A) Predicted cleavage sites with a probability of greater than 75\% for trypsinlike serine proteases in FRETS-VWF73 substrate (highlighted $K$ and $R$ residues) and the cleavage site for ADAMTS13 (indicated by arrow). (B) FRETS-VWF73 substrate conversion in the presence of a concentration series of streptokinase (SK) activated plasminogen (PLG). As a positive control, FRETS-VWF73 substrate conversion by plasma ADAMTS13 is shown (indicated by plasma).(C) FRETS-VWF73 substrate functionality towards plasma ADAMTS13 was tested after pre-exposure to plasmin, which was terminated with inhibitor cocktail prior to analysis (grey circles). In control experiments, we found that inhibitor cocktail protects the substrate from plasmin-mediated destruction (black circles). (B,C) Initial rates of fluorescence as mean \pm standard deviation (SD) of three separate experiments, performed in duplicate. (B) Statistical analysis was performed with Friedman's test with Dunn's multiple comparison test where all values are compared with $0 \mu \mathrm{g} / \mathrm{mL}$ plasmin. (C) Statistical analysis was performed with Wilcoxon matched-pairs signed-rank test. Results were considered statistically significant when $p \leq 0.05$ (ns, non-significant; ${ }^{*} p$ $\leq 0.05$; $^{* *} p \leq 0.01$ ). 
United States); goat polyclonal anti-ADAMTS13 (A300391A; Bethyl, Montgomery, Texas, United States); goat polyclonal anti-plasminogen (GAPG-AP; Affinity Biologicals, Ancaster, ON, Canada); donkey-anti-goat IR dye 800 (92632214; LI-COR, Lincoln, Nebraska, United States); goat-antirabbit Alexa Fluor 680 (A21076; Thermo Fisher Scientific, Waltham, Mississippi, United States); streptokinase (CSL Behring, Marburg, Germany); aprotinin (Sigma-Aldrich, St Louis, Missouri, United States); PPACK (D-PhenylalanineProline-Arginine Chloromethyl Ketone; Haemtech, Essex Junction, Vermont, United States); hirudin (Pharmion, Tiel, the Netherlands); bovine serum albumin (BSA; SigmaAldrich) and purified VWF (Biotest AG, Dreieich, Germany). Plasminogen was purified from plasma as previously described. $^{6}$

\section{In Silico Prediction of Cleavage Sites}

Cleavage sites for trypsinlike serine proteases in FRETSVWF73 substrate were predicted with ExPASy PeptideCutter software. $^{7}$

\section{Direct Effects of Plasmin on FRETS-VWF73 Substrate}

We investigated the influence of plasmin on FRETS-VWF73 substrate in a two-stage experimental set-up. FRETS-VWF73 substrate was dissolved at a concentration of $100 \mu \mathrm{M}$ in $25 \%$ (v/v) dimethyl sulfoxide (DMSO) and diluted to $4 \mu \mathrm{M}$ in assay buffer (5-mM Bis-Tris, $25 \mathrm{mM} \mathrm{CaCl}, 0.005 \%$ Tween-20, $\mathrm{pH}$ 6.0) prior to experiments. Plasminogen $(1.2 \mathrm{mg} / \mathrm{mL})$ was activated with streptokinase $(50 \mathrm{U} / \mathrm{mL})$ for 30 minutes at $37^{\circ} \mathrm{C}$ in HEPES-buffered saline (HBS; 10-mM HEPES, $150-\mathrm{mM} \mathrm{NaCl}, \mathrm{pH} 7.4$ ).

Stage 1: To investigate whether plasmin cleaves between the fluorophore and quencher of FRETS-VWF73 substrate, we incubated 0.2-nmol FRETS-VWF73 in 30- $\mu \mathrm{L}$ assay buffer with $20-\mu \mathrm{L}$ streptokinase-activated plasminogen in HBS. To prevent coagulation of plasma at a later stage in the experiment, $100 \mathrm{U} / \mathrm{mL}$ hirudin (final concentration) was added. The resulting $\mathrm{pH}$ of these $50-\mu \mathrm{L}$ reactions was 6.85 . Buffer controls without streptokinase-activated plasminogen were included in the experiments. Generation of fluorescence was monitored for 10 minutes at $30^{\circ} \mathrm{C}$ in a $96-$ well white MaxiSorp microtiter plate (Thermo Scientific, Roskilde, Denmark) on a SpectraMax $\mathrm{M}^{2}$ fluorescence microplate reader (MTX Laboratory Systems, Bradenton, Florida, United States) equipped with a $340-\mathrm{nm}$ excitation filter and $450-\mathrm{nm}$ emission filter. The reaction rate was calculated by linear regression analysis using PRISM software (version 7.02; GraphPad, San Diego, California, United States).

Stage 2: We next assessed whether FRETS-VWF73 substrate remains sensitive to plasma ADAMTS13 after exposure to plasmin. After following the procedure of stage 1, we subsequently added $10 \mu \mathrm{L}$ of inhibitor cocktail in HBS to all samples $(100-\mu \mathrm{M}$ PPACK, $250 \mathrm{KIU} / \mathrm{mL}$ aprotinin; final inassay concentrations) and incubated for 10 minutes at $30^{\circ} \mathrm{C}$ to block plasmin activity. Subsequently, $30 \mu \mathrm{L}$ of assay buffer was added. Finally, $10 \mu \mathrm{L}$ of diluted plasma in HBS $(20 \% \mathrm{v} / \mathrm{v})$ was added to initiate the assay. In this way, the ratio between assay buffer and HBS (and resulting pH of 6.85) was main- tained between the two stages of the experiment. Generation of fluorescence was monitored for 1 hour at $30^{\circ} \mathrm{C}$ on the SpectraMax $\mathrm{M}^{2}$ fluorescence microplate reader.

\section{Effects of Plasmin on ADAMTS13 Activity}

The direct influence of plasmin on ADAMTS13 activity in plasma was studied by activating plasminogen in $100-\mu \mathrm{L}$ plasma by adding $25-\mu \mathrm{L}$ streptokinase in $\mathrm{HBS}(1,000 \mathrm{U} / \mathrm{mL}$; final concentration) or HBS (buffer control) for 30 minutes at $37^{\circ} \mathrm{C}$. Next, ADAMTS 13 activity was measured by FRETSVWF73 assay as published with minor modifications. ${ }^{5}$ In brief, plasma was diluted in assay buffer $(2 \% \mathrm{v} / \mathrm{v}$, final dilution) and FRETS-VWF73 substrate $(0.2 \mathrm{nmol})$ was added to initiate the assay and fluorescence was measured for 1 hour at $30^{\circ} \mathrm{C}$. The reaction volume of this experiment consists for $97.5 \%$ of assay buffer ( $\mathrm{pH} 6.0$ ), $2 \%$ plasma and $0.5 \%$ HBS (accommodates streptokinase). In further experiments, plasmin activity was inhibited prior to determination of plasma ADAMTS13 activity by addition of $10 \%(\mathrm{v} / \mathrm{v})$ inhibitor cocktail in HBS and $100 \mathrm{U} / \mathrm{mL}$ hirudin (final concentration) and incubation for 10 minutes at $37^{\circ} \mathrm{C}$. Next, $2 \%$ $(\mathrm{v} / \mathrm{v})$ plasma in assay buffer supplemented with inhibitor cocktail was incubated with FRETS-VWF73 substrate (0.2 $\mathrm{nmol}$ ) to initiate the assay. Fluorescence was measured for 1 hour at $30^{\circ} \mathrm{C}$. The reaction volume of this experiment consists for $90 \%$ of assay buffer (pH 6.0), 8\% HBS (accommodates streptokinase and inhibitor cocktail) and $2 \%$ plasma.

\section{Plasmin Activity Assay}

In control experiments, we confirmed activation and inhibition of plasma purified plasminogen under the conditions as described in 'direct effects of plasmin on FRETS-VWF73 substrate' and of plasma plasminogen under the conditions as described in the section 'Effects of Plasmin on Adamts13 Activity'. Hereto, samples were diluted 20-fold in HBS in 96well white MaxiSorp plates. Where indicated, HBS was supplemented with inhibitor cocktail to demonstrate efficacy of plasmin inhibition. A final concentration of $12.5-\mu \mathrm{M}$ I-1390 (plasmin substrate) was added and fluorescence was measured at $37^{\circ} \mathrm{C}$ with excitation at $380 \mathrm{~nm}$ and emission at $460 \mathrm{~nm}$. Initial reaction rates were calculated by linear regression analysis.

\section{SDS-PAGE and Immunoblotting}

Plasminogen, ADAMTS13 and VWF were investigated by immunoblotting $0.5 \mu \mathrm{L}$ of plasma in which plasminogen had been activated and inhibited as described in the section 'Effects of Plasmin on ADAMTS13 Activity'. Plasma samples were diluted 43-fold in sample buffer (62.3-mM Tris- $\mathrm{HCl}$ [pH 6.8], $10 \%$ glycerol, $2 \%$ SDS, $0.001 \%$ bromophenol blue) and supplemented where indicated with $8.3-\mathrm{mM}$ dithiothreitol (DTT) for reduction. Samples were boiled at $95^{\circ} \mathrm{C}$ for $10 \mathrm{~min}-$ utes and $30 \mu \mathrm{L}$ (containing $0.5 \mu \mathrm{L}$ of original plasma) was separated by SDS-PAGE on $7.5 \%$ gels. For separation of ADAMTS13 and plasminogen under reducing conditions, 4 to $12 \%$ gradient gels were used. Proteins were transferred to immobilon-FL polyvinylidene fluoride (PVDF) membrane (Merck Millipore, Tullagreen, Ireland) and blocked with 1:1 
odyssey blocking buffer (LI-COR) and Tris-buffered saline (TBS; 10-mM Tris, 150-mM NaCl, pH 7.4), followed by overnight incubation with the anti-ADAMTS13, anti-plasminogen or anti-VWF, diluted $1: 2,500$ at $4^{\circ} \mathrm{C}$. The anti-ADAMTS13 antibody is affinity purified using an epitope specific to ADAMTS13 mapping between residues 250 and 350 (part of the metalloproteinase and disintegrin domain) using the numbering given in entry NP_620594.1 (GeneID 11093). Membranes were subsequently incubated with donkeyanti-goat IR dye 800 (for anti-ADAMTS13 and anti-plasminogen) or goat-anti-rabbit Alexa Fluor 680 antibody (for antiVWF) diluted 1:10.000 for 2 hours at room temperature (RT). Membranes were scanned on a near-infrared scanner (Odyssey) and band intensities were quantified with Odyssey V3.0 software (LI-COR).

\section{ADAMTS13 Binding to Immobilized VWF}

Binding of ADAMTS13 to immobilized VWF in control and streptokinase activated and inhibited plasma (prepared as described in the section 'Effects of Plasmin on ADAMTS13 Activity') was investigated as published with minor modifications. ${ }^{8}$ Ninety-six-wells Nunc-Immuno MaxiSorp microtiter plates (Thermo Scientific) were coated with VWF overnight at RT $(100 \mu \mathrm{L} /$ well; $10 \mu \mathrm{g} / \mathrm{mL}$ VWF in phosphate buffered saline [PBS; $137-\mathrm{mM} \mathrm{NaCl}, 2.7-\mathrm{mM} \mathrm{KCl}, 9.2-\mathrm{mM}$ $\left.\left.\mathrm{Na}_{2} \mathrm{HPO}_{4} \cdot \mathrm{H}_{2} \mathrm{O}, 1.76-\mathrm{mM} \mathrm{KH} \mathrm{KPO}_{4}, \mathrm{pH} 7.4\right]\right)$ and blocked with $200 \mu \mathrm{L} /$ well blocking buffer (PBS, 2.5\% BSA, 0.1\% Tween20 ) for 1 hour at RT. Where indicated, plasmin activity was triggered and terminated in plasma samples prior to binding experiments (as described in the section 'Effects of Plasmin on ADAMTS13 Activity'), buffer controls were included to correct for sample dilution. All plasma samples were supplemented with 5-mM ethylenediaminetetraacetic acid (EDTA; to block ADAMTS13 activity). A sample of $100 \mu \mathrm{L}$ was incubated in each well for 2 hours at RT. Next, the wells were washed three times with PBS $0.1 \%$ Tween- 20 and bound ADAMTS13 was eluted in three times the sample buffer. The eluted ADAMTS13 from 48 wells per condition was combined and equally divided into a non-reduced, and a reduced sample (to which DTT was added) prior to analyses.

\section{Patient Plasma Samples}

Citrated plasma was collected from systemic amyloidosis patients and controls without systemic amyloidosis (i.e., localized amyloidosis) at Groningen University Medical Center under institutional ethical approval and informed consent as described earlier. ${ }^{9,10}$ Selection was based on positive Congo red biopsies and number and location of sites with amyloid depositions. In a previous study, plasmin- $\alpha 2$ antiplasmin (PAP) complex levels were determined in duplicate within 5 months using a commercially available ELISA (Technoclone GmbH, Vienna, Austria, Cat. No. TC11060), according to the manufacturer's instructions. ${ }^{11}$ ADAMTS13 activity was determined as described earlier. The binding of ADAMTS13 in patient plasma to immobilized VWF was investigated by incubating $30-\mu \mathrm{L}$ plasma samples (undiluted) for more than 2 hours on immobilized VWF. Per patient six wells were combined and analysed on western blot. Band intensities of uncleaved 190-kDa and truncated 170-kDa ADAMTS13 products were quantified by densitometry with Odyssey V3.0 software (LI-COR). The fraction of truncated ADAMTS13 was expressed relative to the fraction of uncleaved ADAMTS13 in individual patients.

\section{Statistical Analysis}

Statistical analysis was performed with PRISM software (version 7.02; GraphPad) using Wilcoxon matched-pairs signed rank test or Friedman test with Dunn's multiple comparison test. Correlation was tested using non-parametric Spearman's correlation. Results were statistically significant when $p \leq 0.05$.

\section{Results}

\section{The ADAMTS13 Binding Tail of FRETS-VWF73 Substrate Is Sensitive to Plasmin Cleavage}

ExPASy PeptideCutter software predicts that the FRETSVWF73 sequence contains five cleavage sites for trypsinlike enzymes. ${ }^{7}$ Four of these are located in the sequence that mediates ADAMTS13 binding (-Fig. 1A), but no cleavage sites are predicted between the fluorophore and quencher.

We exposed FRETS-VWF73 substrate to different concentrations of purified streptokinase-activated plasminogen (plasmin activity was confirmed in -Supplementary Figure S1 (online only)). We found that no fluorescence was generated (-Fig. 1B), confirming the sequence between fluorophore and quencher does not contain a plasmin cleavage site. $^{12,13}$ When FRETS-VWF73 was exposed to plasmin activity for 10 minutes and subsequently blocked with inhibitor cocktail (efficacy confirmed in -Supplementary Figure S1B (online only)), its functionality to act as a substrate for plasma ADAMTS13 was dose dependently impaired (-Fig. 1C; grey circles). The inhibitor cocktail protects the substrate from plasmin-mediated destruction, which eliminates potential analytical threats in our next experiments (-Fig. 1C; black circles).

\section{Plasmin Predominantly Cleaves ADAMTS13 in Plasma under Static Conditions}

We next triggered plasmin activity in plasma with streptokinase (confirmed in -Supplementary Figure S2 (online only)), terminated its activity with inhibitor cocktail and investigated VWF and ADAMTS13 by immunoblotting under reduced conditions. We found that VWF monomer content did not significantly diminish after plasmin activity (-Fig. 2A and B), although a 176-kDa cleavage product appeared. ${ }^{4}$ This indicates that globular VWF is largely resistant against plasmin cleavage, which fits well with the earlier-reported shear-dependent cleavage by us ${ }^{2}$ and others. ${ }^{12}$ Under the same conditions, the ADAMTS13 band at $190 \mathrm{kDa}$ was consumed $(-78.7 \pm 13.2 \%, p=0.0039 ;-$ Fig. 2 C and $\mathbf{D})$ and there was some evidence for the generation of truncated products at approximately 130 and $90 \mathrm{kDa}$ (-Fig. 2C). These findings together show that under static conditions in plasma, plasmin favours cleavage of ADAMTS13 over globular VWF. 



Fig. 2 Plasmin predominantly cleaves ADAMTS13 (a disintegrin and metalloproteinase with a thrombospondin type 1 motif, member 13) in plasma under static conditions. (A-D) The effect of plasmin on von Willebrand factor (VWF) and ADAMTS13 in plasma was investigated by triggering plasminogen (PLG) activation with streptokinase (SK; $1,000 \mathrm{U} / \mathrm{mL}, 30$ minutes at $37^{\circ} \mathrm{C}$ ), followed by addition of inhibitor


terminate plasmin activity. As a control, plasma was incubated with buffer without streptokinase and inhibited with inhibitor cocktail as before. $(A, C)$ Plasma of $0.5 \mu \mathrm{L}$ was separated per lane by SDS-PAGE and immunoblotted for VWF (A), and ADAMTS13 (C), under reduced conditions. Representative immunoblots are shown. (B,D) Quantification of band densities of multiple experiments $(n=9)$ presented as median \pm interquartile range. Statistical analysis was performed with Wilcoxon matched-pairs signed-rank test (average band density of three control samples per experiment was set to $100 \%)$. Results were considered statistically significant when $p \leq 0.05$ (ns, non-significant; $\left.{ }^{* *} p \leq 0.01\right)$.

\section{Plasmin Cleavage Enhances ADAMTS13 Activity in Plasma}

It was previously reported that plasmin cleaves recombinant ADAMTS13. ${ }^{3}$ In a buffered system, this cleavage is extensive and ultimately results in ADAMTS13 inactivation. ${ }^{3}$ It is noteworthy that the first stages of plasmin cleavage take place at the C-terminus, resulting in truncated products with an intact metalloprotease domain. In hyperfibrinolytic ( $\alpha 2-$ antiplasmin deficient) plasma, cleavage of recombinant ADAMTS13 by plasmin takes place much less rapidly than in a buffered system, but identical C-terminally truncated products are formed first. ${ }^{13}$ It was suggested that these fragments were no longer active.

During our next experiments, we made a striking observation. When plasminogen activation was triggered in plasma, FRETS-VWF73 conversion was increased by $21.6 \pm 6.6 \%$, compared with buffer control plasma samples $(p=0.0313 ; \quad$-Fig. 3A). In our previous experiment (-Fig. 1B), we had found that plasmin does not elicit
FRETS-VWF73 substrate fluorescence in a buffered system. This led us to hypothesize that plasmin indirectly contributes to increased FRETS-VWF73 conversion in plasma. To investigate this, we modified the FRETS-VWF73 assay into a twostep procedure. First, plasmin activity was triggered. Second, inhibitor cocktail was added to reduce plasmin activity to less than 1\% (-Fig. 3B). When FRETS-VWF73 conversion was determined in plasma in which plasmin had been active, we consistently detected an increase in substrate conversion compared with buffer control plasma samples $(21.2 \pm 6.9 \%$, $p=0.0313 ;$ - Fig. 3C). This indicates that, although plasmin cleaves ADAMTS13, its apparent activity increases. Next, we set out to identify the responsible mechanism.

\section{Plasmin Generates C-Terminally Truncated ADAMTS13 Products with VWF-Binding Properties}

The interaction between the spacer domain of ADAMTS13 and the A2 domain of (unfolded) VWF is important for efficient VWF cleavage. A recent study elegantly demonstrates that the C-terminal complement $\mathrm{C} 1 \mathrm{r} / \mathrm{C} 1 \mathrm{~s}$, Uegf, Bmp1 (CUB) domains of ADAMTS13 limit its function by intramolecular binding to the spacer domain. ${ }^{14}$ The resulting 'closed' conformation can be opened by binding to globular VWF, unlocking the full activity of ADAMTS13. Interestingly, this same study, as well as an earlier study, ${ }^{15}$ showed that recombinant ADAMTS13 variants lacking either the C-terminal tail or the CUB domains displayed an increased activity without initial binding to globular VWF.

In our next experiments, we investigated the effect of plasmin on the VWF-binding properties of ADAMTS13. Hereto, we incubated plasma samples on immobilized VWF in microtiter plates. After washing, bound ADAMTS13 was eluted with sample buffer and analysed by western blotting with an antibody against the metalloprotease domain. ${ }^{8}$ In plasma, the major product that is captured is $190 \mathrm{kDa}$ (-Fig. 4A and B; reducing conditions). A second minor product is seen at approximately $170 \mathrm{kDa}$. When plasmin activity is triggered and terminated in plasma before incubation on immobilized VWF, less full-length protein (190 kDa) binds. In this case, multiple truncated species of approximately 170, 130 and $90 \mathrm{kDa}$ are captured. ADAMTS13 contains intramolecular disulphide linkages; therefore, these fragments could remain covalently associated under nonreducing conditions. Analyses under non-reducing conditions show a highly similar picture: intact ADAMTS13 is captured from plasma before, but much less after exposure to plasmin. Instead, truncated variants that contain the protease domain are captured ( $\mathbf{F i g . ~ 4 C}$ and $\mathbf{D}$ ).

\section{Truncated ADAMTS13 in Systemic Amyloidosis}

Patients with systemic amyloidosis are in a chronic hyperfibrinolytic state, which may contribute to their reduced haemostatic potential and bleeding problems. ${ }^{16}$ We investigated ADAMTS13 in these patients and control samples $(n=11$ and 8 , respectively), of which PAP had been previously determined. In line with our earlier report, PAP complex levels differed significantly between controls (median; $171.5 \mathrm{ng} / \mathrm{mL}$; interquartile range [IQR] 130.8-221.8 ng/mL) and patients 
A

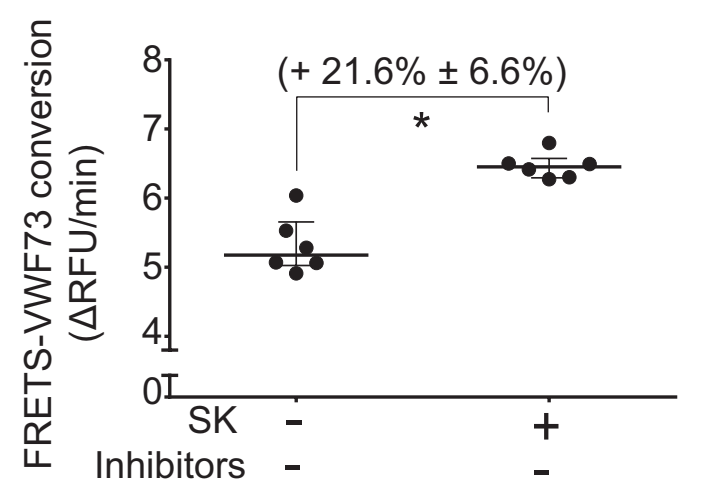

C
B

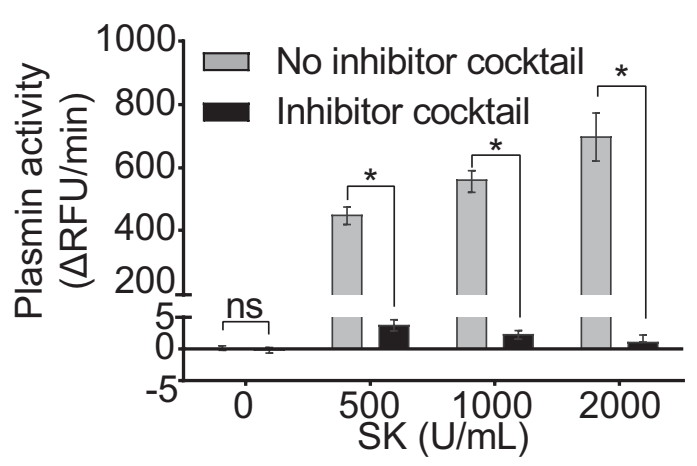

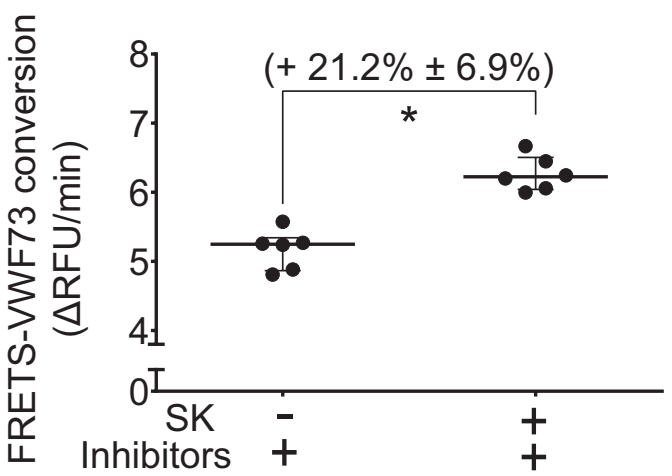

(median; 1,238 ng/mL; IQR 475.8-4103 ng/mL; - Fig. 5A). We next investigated ADAMT13 truncation in these patient plasmas. As before, we analysed ADAMTS13 binding to immobilized VWF. In our earlier experiments (-Fig. 4A), plasmin generates a 170-kDa C-terminally truncated ADAMTS13. To a minor extent, this product is already present in normal plasma (-Fig. 4A). We found that this 170-kDa band was represented more strongly in plasma of patients with systemic amyloidosis than of controls (- Fig. 5B shows a representative blot). To correct for inter-individual differences, we expressed truncated 170-kDa ADAMTS13 relative to 190-kDa ADAMTS13 for each plasma sample. The presence of truncated $170-\mathrm{kDa}$ ADAMTS13 correlates with the extent of fibrinolytic activity (i.e., PAP complexes; $r=0.5491, p=0.0149$; - Fig. 5C). These findings were not dependent on the patient with the highest PAP complex levels and accompanying ADAMTS13 truncation ( $r=0.4696, p=0.0493$ after exclusion). ADAMTS13 activity in these samples was determined with the FRETS-VWF73 assay (-Fig. 5D), but no significant difference was found between the control and patient groups. We observed greater than 20\% variation in ADAMTS13 activity between various plasma samples, which is in good correspondence to earlier reports. ${ }^{5}$ This may obscure increased activity as a function of plasmin-mediated truncation.

\section{Discussion}

We here report the finding that plasmin under static conditions in plasma rapidly truncates ADAMTS13 and this is accompanied by increased ADAMTS13 activity in the FRETS-VWF73 assay. Moreover, multiple truncated forms of ADAMTS13 retain the capacity to bind to VWF.

In our study, we ruled out that plasmin is responsible for the observed increased FRETS-VWF73 substrate fluorescence. In contrast, we found that plasmin destroys FRETS-VWF73 substrate in a buffered system. Although this effect is modest in 
A

Captured ADAMTS13
reduced $(7.5 \%)$

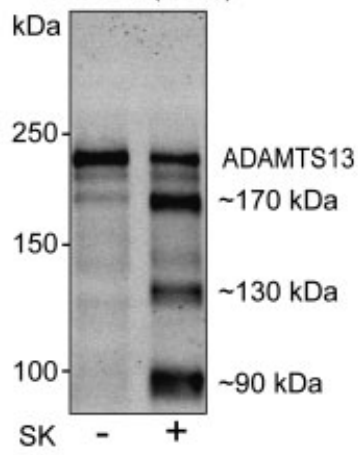

C

Captured ADAMTS13

non-reduced $(7.5 \%)$

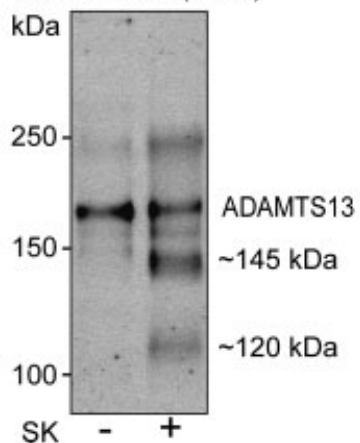

B

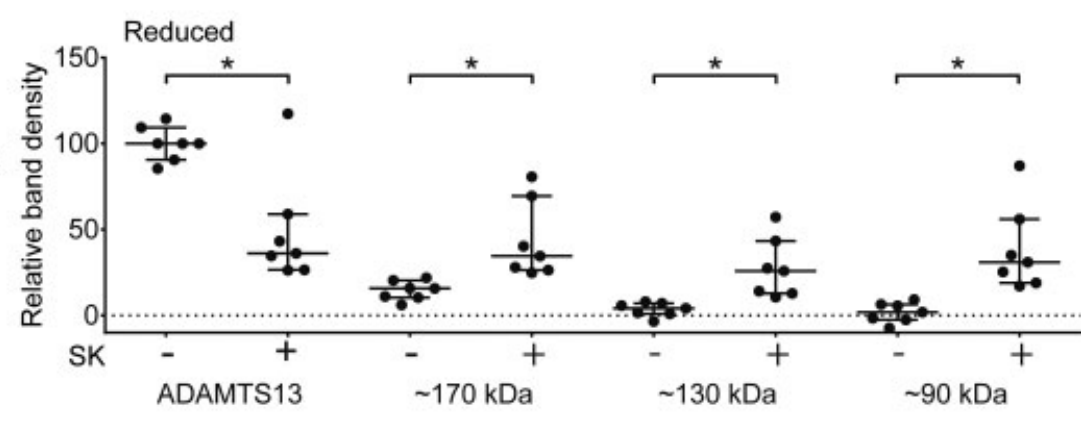

D

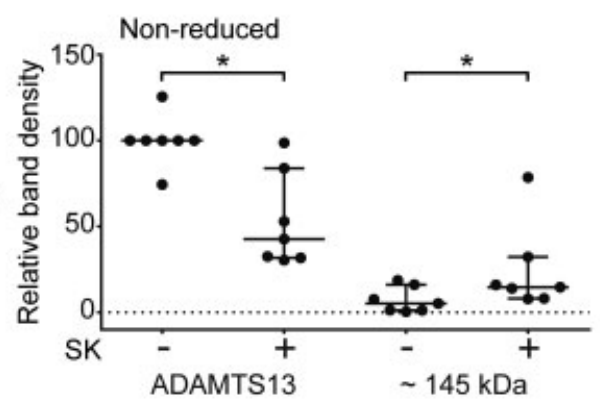

Fig. 4 Plasmin generates truncated ADAMTS13 (a disintegrin and metalloproteinase with a thrombospondin type 1 motif, member 13) variants with VWF-binding properties and a metalloprotease domain. (A-D) The binding of plasma ADAMTS13 to immobilized VWF was analysed by immunoblotting with an antibody against the metalloprotease domain under reducing $(A, B)$ and non-reducing conditions (C,D). Plasmin activity was transiently triggered with the two-stage approach in which plasma plasminogen is activated with streptokinase and subsequently inhibited with inhibitor cocktail prior to the incubation of the plasma samples on immobilized VWF (in presence of ethylenediaminetetraacetic acid [EDTA]). As a control, buffer without streptokinase was included. (A-D) Representative immunoblots and quantification of band densities from repeated experiments (median \pm interquartile range, $n=7$ ). Statistical analysis was performed with Wilcoxon matched-pairs signed-rank test. Results were considered statistically significant when $p \leq 0.05\left({ }^{*} p \leq 0.05\right)$.

normal plasma that contains the powerful plasmin inhibitor $\alpha 2$-antiplasmin, it might generate an analytic threat in plasma samples with partial ${ }^{2}$ or complete ${ }^{13} \alpha 2$-antiplasmin deficiency. If needed, this potential analytical threat can be averted with the use of plasmin-blocking serine protease inhibitors, as we used in this study.

Plasmin can cleave ADAMTS13 in buffer at multiple positions: at Arg-257/Ala-258 (metalloprotease domain), at Arg888/Thr-889 (linker region between the fourth and fifth thrombospondin type 1 repeat [TSP-1]) and at Arg-1176/ Arg-1177., ${ }^{37}$ It is likely that similar cleavage events take place during plasminogen activation in plasma, corresponding to the truncated ADAMTS13 products that can bind to immobilized VWF (-Fig. 4A and B). Intramolecular disulphide linkage assures covalent association of the fragments generated by cleavage at Arg-257/Ala258 or Arg-88/Thr889. ${ }^{3,18}$ However, C-terminal truncation after cleavage at Arg-1176/Arg-1177 truncates ADAMTS13 by removal of the eighth TSP- 1 and both CUB domains. ${ }^{17,18}$ This is consistent with the capture of truncated ADAMTS13 under nonreduced conditions ( $\mathbf{- F i g .} \mathbf{4 C}$ and $\mathbf{D}$ ).
Based on our findings, we propose that monomeric ADAMTS13 is C-terminally truncated by plasmin. However, it is noteworthy that we observed a second plasmin-sensitive ADAMTS13-immunoreactive high-molecular weight product $(\sim 350 \mathrm{kDa})$ in plasma (-Supplementary Figure S3 (online only)). Future studies will have to determine whether this species of ADAMTS13 contributes to the observed increased ADAMTS13 activity after plasminogen activation.

When ADAMTS13 is not 'in action', its CUB domains interact with its spacer region to ensure a closed conformation. During VWF binding, these CUB domains interact with VWF. ${ }^{19}$ This causes ADAMTS13 to change conformation, which increases its activity ('conformational activation'). ${ }^{14}$ We hypothesize that plasmin-mediated removal of CUB domains causes ADAMTS13 to adopt an open conformation (resembling conformational activation ${ }^{14}$ ), with increased accessibility of the spacer domain for unfolded VWF.

We propose that our findings reflect a physiological mechanism to enhance ADAMTS13 activity. When the 

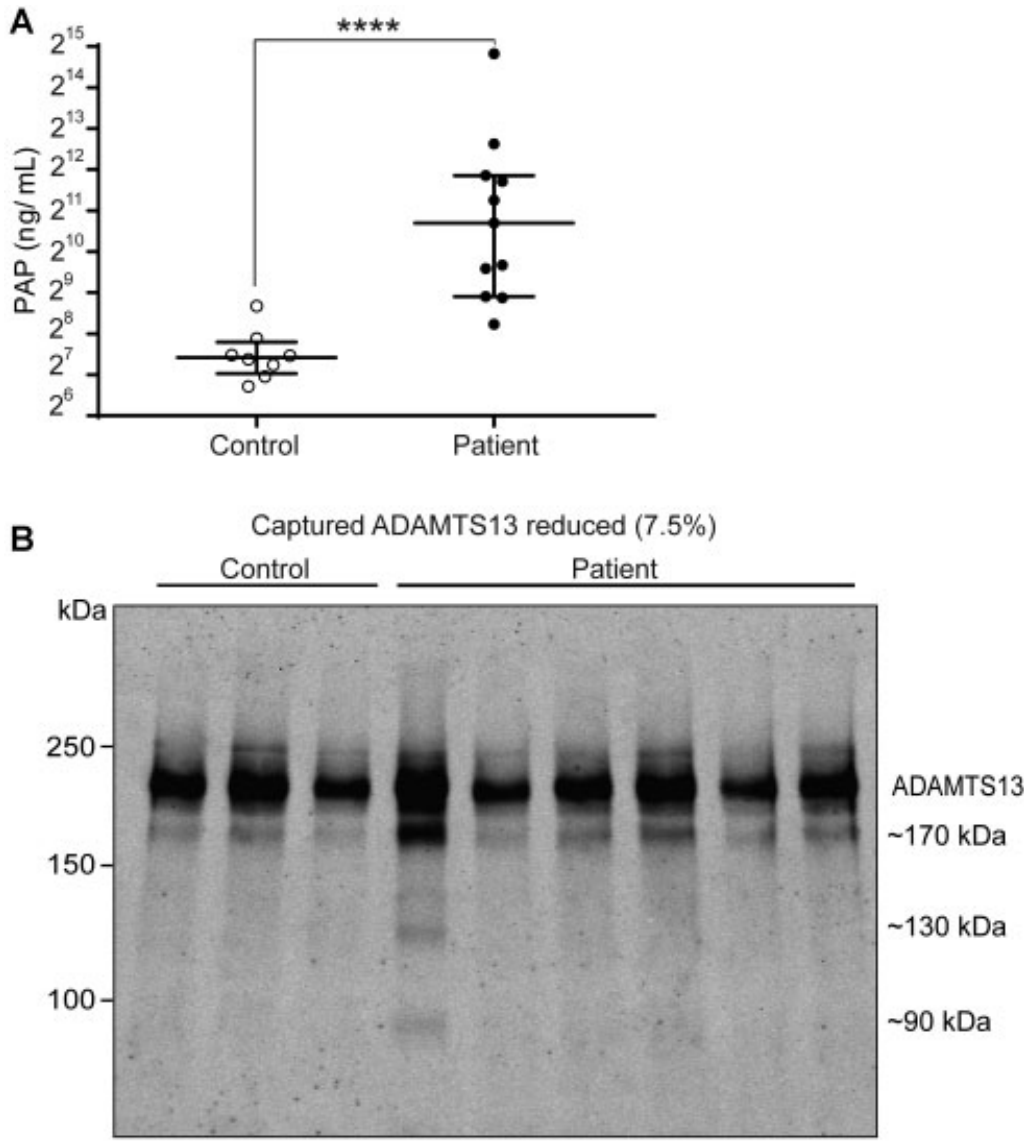

C

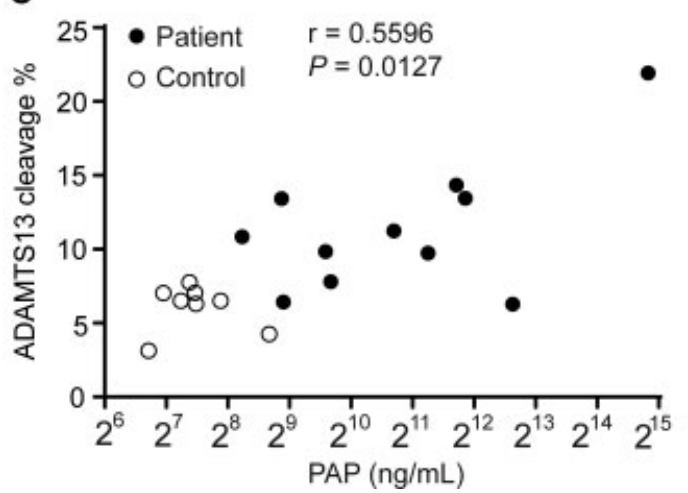

D

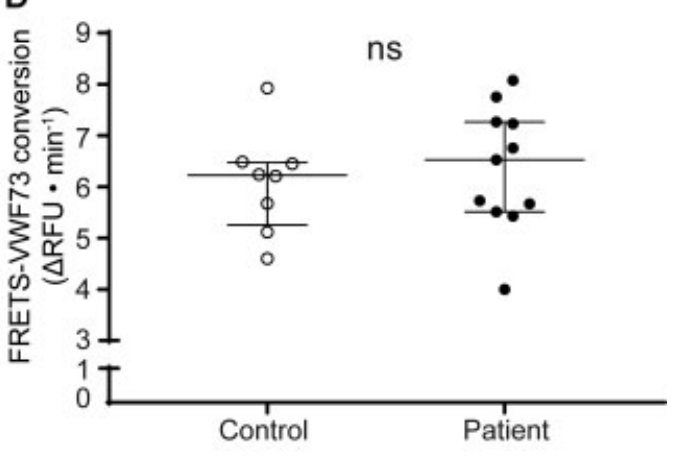

Fig. 5 Truncated ADAMTS13 (a disintegrin and metalloproteinase with a thrombospondin type 1 motif, member 13) in systemic amyloidosis. (A) Plasmin- $\alpha 2$-antiplasmin complex levels (PAP) in systemic amyloidosis patients and controls without systemic amyloidosis $(n=11$ and 8 , respectively). Plasma samples were incubated on immobilized VWF for ADAMTS13 binding (in presence of EDTA). (B) Bound ADAMTS13 was analysed by immunoblotting. (C) Correlation plot of PAP complex levels and truncated ADAMTS13 (170/190 kDa ADAMTS13 ratio) of these samples $(r=0.5491, p=0.0149)$. (D) ADAMTS13 activity of systemic amyloidosis patients and controls, measured with the FRETS VWF73 assay. (A,D) Statistical analysis was performed with Wilcoxon matched-pairs signed-rank test. (B) Representative immunoblot. (C) Correlation was tested using non-parametric Spearman correlation. Results were considered statistically significant when $p \leq 0.05$ (ns, non-significant, $\left.{ }^{* * * *} p \leq 0.0001\right)$.

microvasculature senses hypoxia, as may occur during microthrombosis, plasmin is locally generated on endothelial cells. Besides its direct effects on $\mathrm{VWF}^{4,20}$ and plateletVWF complexes, ${ }^{2}$ plasmin cleaves ADAMTS13, leading to enhanced activity. It is attractive to hypothesize that similar truncation of ADAMTS13 and associated increased activity contributes to the haemorrhagic phenotype in hyperfibrinolytic disorders, such as systemic amyloidosis.

\section{Conclusion}

Our findings indicate that under static conditions in plasma, plasmin cleaves ADAMTS13 more rapidly than VWF. C-terminally truncated ADAMTS13 can bind to immobilized VWF and is associated with an enhanced activity. Matching Cterminally truncated ADAMTS13 forms are generated in vivo during increased fibrinolytic activity. 


\section{What is known about this topic?}

- Plasmin can cleave both von Willebrand factor (VWF) and ADAMTS13 (a disintegrin and metalloproteinase with a thrombospondin type 1 motif, member 13); this is assumed to have opposite consequences in haemostasis.

- ADAMTS13 circulates in a closed conformation because of intramolecular self-association between its C-terminal $\mathrm{C} 1 \mathrm{r} / \mathrm{C} 1 \mathrm{~s}$, Uegf, Bmp1 (CUB) domain and centrally positioned spacer domain.

- ADAMTS13 undergoes conformational changes when it binds to VWF, increasing its activity ("conformational activation").

\section{What does this paper add?}

- Plasmin cleaves ADAMTS13 more rapidly than VWF under static conditions in plasma. This results in functional C-terminally truncated forms of ADAMTS13 that can bind to immobilized VWF.

- ADAMTS13 activity in plasma increases by approximately 20\% during or after exposure to plasmin activity.

- Increased levels of C-terminally truncated ADAMTS13 are present in plasma of patients with systemic amyloidosis, and correlates with their hyperfibrinolytic state.

\section{Author Contributions}

C.C. Clark, M.M. Mebius, S.d. Maat and C. Maas designed and performed research, analysed data and wrote the manuscript. B.P.C. Hazenberg, A.G.M. Tielens, P.G.d. Groot, R.T. Urbanus and J.J.v. Hellemond contributed vital new reagents and analytical tools. C.C. Clark and Mirjam M. Mebius contributed equally.

Conflicts of Interest

The authors declare no competing financial interests.

\section{Financial Support}

CM gratefully acknowledges financial support from the Landsteiner Foundation for Blood Transfusion Research and the Netherlands Thrombosis Foundation.

\section{References}

1 Kremer Hovinga JA, Coppo P, Lämmle B, Moake JL, Miyata T, Vanhoorelbeke K. Thrombotic thrombocytopenic purpura. Nat Rev Dis Primers 2017;3:17020
2 Tersteeg C, de Maat S, De Meyer SF, et al. Plasmin cleavage of von Willebrand factor as an emergency bypass for ADAMTS13 deficiency in thrombotic microangiopathy. Circulation 2014;129(12): 1320-1331

3 Crawley JTB, Lam JK, Rance JB, Mollica LR, O'Donnell JS, Lane DA. Proteolytic inactivation of ADAMTS13 by thrombin and plasmin. Blood 2005;105(03):1085-1093

4 Berkowitz SD, Dent J, Roberts J, et al. Epitope mapping of the von Willebrand factor subunit distinguishes fragments present in normal and type IIA von Willebrand disease from those generated by plasmin. J Clin Invest 1987;79(02):524-531

5 Kokame K, Nobe Y, Kokubo Y, Okayama A, Miyata T. FRETSVWF73, a first fluorogenic substrate for ADAMTS13 assay. Br J Haematol 2005;129(01):93-100

6 de Maat S, Björkqvist J, Suffritti C, et al. Plasmin is a natural trigger for bradykinin production in patients with hereditary angioedema with factor XII mutations. J Allergy Clin Immunol 2016;138 (05):1414-1423.e9

7 Wilkins MR, Gasteiger E, Bairoch A, et al. Protein identification and analysis tools in the ExPASy server. Methods Mol Biol 1999; 112:531-552

8 Majerus EM, Anderson PJ, Sadler JE. Binding of ADAMTS13 to von Willebrand factor. J Biol Chem 2005;280(23):21773-21778

9 Maas C, Govers-Riemslag JW, Bouma B, et al. Misfolded proteins activate factor XII in humans, leading to kallikrein formation without initiating coagulation. J Clin Invest 2008;118(09): 3208-3218

10 Hazenberg BP, van Rijswijk MH, Piers DA, et al. Diagnostic performance of ${ }^{123}$ I-labeled serum amyloid P component scintigraphy in patients with amyloidosis. Am J Med 2006;119(04):355. e15-355.e24

11 Bouma B, Maas C, Hazenberg BPC, Lokhorst HM, Gebbink MF. Increased plasmin- $\alpha 2$-antiplasmin levels indicate activation of the fibrinolytic system in systemic amyloidoses. J Thromb Haemost 2007;5(06):1139-1142

12 Brophy TM, Ward SE, McGimsey TR, et al. Plasmin cleaves von Willebrand factor at K1491-R1492 in the A1-A2 linker region in a shear- and glycan-dependent manner in vitro. Arterioscler Thromb Vasc Biol 2017;37(05):845-855

13 Feys HB, Vandeputte N, Palla R, et al. Inactivation of ADAMTS13 by plasmin as a potential cause of thrombotic thrombocytopenic purpura. J Thromb Haemost 2010;8(09):2053-2062

14 South K, Luken BM, Crawley JT, et al. Conformational activation of ADAMTS13. Proc Natl Acad Sci U S A 2014;111(52):18578-18583

15 Feys HB, Anderson PJ, Vanhoorelbeke K, Majerus EM, Sadler JE. Multi-step binding of ADAMTS-13 to von Willebrand factor. J Thromb Haemost 2009;7(12):2088-2095

16 Hammarström P. The bloody path of amyloids and prions. J Thromb Haemost 2007;5(06):1136-1138

17 Hiura H, Matsui T, Matsumoto M, et al. Proteolytic fragmentation and sugar chains of plasma ADAMTS13 purified by a conformation-dependent monoclonal antibody. J Biochem 2010;148(04): 403-411

18 Lam JK, Chion CK, Zanardelli S, Lane DA, Crawley JT. Further characterization of ADAMTS-13 inactivation by thrombin. J Thromb Haemost 2007;5(05):1010-1018

19 Crawley JT, de Groot R, Xiang Y, Luken BM, Lane DA. Unraveling the scissile bond: how ADAMTS13 recognizes and cleaves von Willebrand factor. Blood 2011;118(12):3212-3221

20 Wohner N, Kovács A, Machovich R, Kolev K. Modulation of the von Willebrand factor-dependent platelet adhesion through alternative proteolytic pathways. Thromb Res 2012;129(04):e41-e46 